\title{
ALTERNATIVAS DE CONTROLE PÓS-COLHEITA DA PODRIDÃO-PARDA E DA PODRIDÃO-MOLE EM FRUTOS DE PESSEGUEIRO ${ }^{1}$
}

\author{
VICENTELUIZDECARVALHO², RODRIGO LUZDACUNHA ${ }^{3}$, \\ NILTON NAGIB JORGE CHALFUN ${ }^{4}$, PEDRO HENRIQUE ABREU MOURA ${ }^{5}$
}

RESUMO - As exigências do consumidor são cada vez maiores com relação à qualidade de produtos in natura. As podridões, além de causar perdas na produção, reduzem a qualidade final do fruto, interferindo significativamente na comercialização. Objetivou-se, com este trabalho, avaliar a eficiência de produtos alternativos, aplicados na pós-colheita, no controle da podridão-parda (Monilinia fructicola) e da podridãomole (Rhizopus spp.) em pêssegos. O experimento foi conduzido no município de Nepomuceno-MG, em talhão de pessegueiro da cultivar Diamante, com 10 anos de idade. O delineamento experimental utilizado foi o inteiramente casualizado, com 7 tratamentos e 3 repetições. Para o estudo, foram selecionados frutos sem lesões e sem sintomas das doenças. Os frutos foram mergulhados por 30 segundos em solução contendo os seguintes tratamentos: 1-Óleo de cravo 0,01\%; 2-Dióxido de cloro 0,1\%; 3-Dióxido de cloro 0,05\%; 4-Cloreto de benzalcônio 0,25\%; 5-Dicloran 0,12\%; 6-Iprodione 0,15\% e 7-Testemunha (somente água). Após os tratamentos, os frutos, em número de 10, foram colocados em bandejas esterilizadas, em três repetições. O experimento foi conduzido em condições de ambiente não controlado, sendo feitas avaliações do desenvolvimento das doenças aos 3 e aos 5 dias após os tratamentos em 2005 e aos 3; 6 e aos 9 dias após o tratamento em 2006. O iprodione controlou a incidência e a severidade de M. fructicola e Rhizopus spp. O dicloran foi o tratamento mais eficiente para o controle do Rhizopus spp. e intermediário para M. fructicola. Os tratamentos com óleo de cravo e dióxido de cloro, na maior dose, reduziram a incidência de Rhizopus spp. e para severidade apresentaram resultados intermediários.

Termos para indexação: pêssego, podridões dos frutos, controle de doenças pós-colheita.

\section{ALTERNATIVES FOR POST-HARVEST CONTROL OF BROWN ROT AND SOFT ROT IN PEACH FRUITS}

\begin{abstract}
The consumers demand are increasing with regard to the quality of in nature products. The rot, besides causing loss in production, reduce the quality of the product, interfering significantly in the marketing. The objective of this study was to evaluate the efficiency of alternative products, used during the post-harvest, in order to control the brown rot (Monilinia fructicola) and the soft rot (Rhizopus spp.) in peaches. The experiment was carried out in the municipality of Nepomuceno-MG in peaches of cultivar Diamond, of 10 years of planting. The experimental design was completely randomized, with 7 treatments and 3 repetitions. For the study, it was selected fruits without injuries and symptoms of the diseases. The fruits were immersed for 30 seconds in a solution containing the following treatments: 1- clove oil 0.01\%; 2Chlorine dioxide $0.1 \%$; 3-Chlorine dioxide $0.05 \%$; 4-Benzalkonium chloride $0.25 \%$; 5-Dichloran $0.12 \%$, 6Iprodione $0.15 \%$ and 7 -control (water only). After the treatments, the fruits, in a number of 10, were placed in sterile trays, in three replicates. The experiment was carried out in uncontrolled environmental conditions, the development of the diseases were evaluated 3 and 5 days after the treatments in 2005 and 3, 6 and 9 days after treatment in 2006. The use of Iprodione controlled the incidence and severity of M. fructicola and Rhizopus spp. The Dichloran was the most effective treatment in the control of Rhizopus spp. and intermediate in the control of $M$. fructicola. The treatments with clove oil and chlorine dioxide, at the highest concentration, reduced the incidence of Rhizopus spp. and to severity they showed intermediate results.
\end{abstract}

Index terms: peaches, fruits rot, post-harvest diseases control.

\footnotetext{
1(Trabalho 126-08). Recebido em: 26-05-2008. Aceito para publicação em: 02-12-2008.

${ }^{2}$ Eng. Agrôn., Mestre em Fitopatologia, Empresa de Pesquisa Agropecuária de Minas Gerais (EPAMIG). E-mail: vicentelc@epamig.ufla.br

${ }^{3}$ Eng. Agrôn., Doutor em Fitotecnia, Empresa de Pesquisa Agropecuária de Minas Gerais (EPAMIG). E-mail: rodrigo@epamig.ufla.br

${ }^{4}$ Eng. Agrôn., Prof. Doutor em Fitotecnia, Universidade Federal de Lavras (UFLA). E-mail: nchalfun@ufla.br

${ }^{5}$ Graduando em Agronomia, Universidade Federal de Lavras (UFLA). Bolsista de Iniciação Científica FAPEMIG/EPAMIG.

E-mail: pedrohamoura@yahoo.com.br
} 


\section{INTRODUÇÃO}

O pêssego, juntamente com outras frutas de caroço, como a nectarina e a ameixa, evidencia, nos últimos anos, notável expansão, tanto da produção, quanto do consumo interno. Atualmente, a área colhida de pêssego no Brasil é da ordem de $30 \mathrm{mil}$ hectares, com a produção anual ultrapassando 100 mil toneladas, destinando-se $57 \%$ para o consumo "in natura" e $43 \%$ para industrialização (Junqueira \& Peetz, 2003).

$\mathrm{O}$ pessegueiro pode ser afetado por diversas doenças, principalmente aquelas provocadas por fungos que causam podridões pós-colheita, que são responsáveis por perdas significativas na qualidade e/ou na quantidade dos produtos agrícolas durante as etapas de comercialização. Essas perdas podem oscilar entre 10 e 50\%, segundo Alvarez \& Nishijima (1987); Wilson et al. (1994) e Benato (1999).

Entre os microrganismos associados às podridões pós-colheita de frutos de pessegueiro e nectarineira, destacam-se os fungos Monilinia fructicola e Rhizopus spp., que são considerados os de maior incidência (Martins \& Amorim, 2005).

A podridão-parda, causada pelo fungo Monilinia fructicola, é considerada a principal doença das rosáceas de caroço, tanto na fase précolheita como na pós-colheita. No Rio Grande do Sul, a doença já foi responsável por perdas de até $25 \%$ dos frutos de pessegueiro destinados à industrialização (Andrade, 1995; Fortes, 2003).

A podridão-mole, causada pelo fungo Rhizopus spp., é uma podridão importante para pessegueiros e ameixeiras, causando sérias perdas nas fases pós-colheita de transporte e comercialização (Andrade, 1995; Bleicher, 1997). O fungo é altamente destrutível, com grande capacidade saprofítica, exigindo ferimentos para sua penetração, após a qual rapidamente coloniza o fruto, causando uma podridão-mole e aquosa, geralmente não incidindo em frutos imaturos, sendo raramente vistos no campo (Alvarez \& Nishijima, 1987).

O controle dessas doenças pós-colheita baseia-se em uma série de medidas preventivas, iniciando em pré-colheita, como sanitização do pomar, e continuando na pós-colheita, incluindo o tratamento químico dos frutos (Fortes \& Bettiol, 1997; Feliciano \& Sachs, 1984; Bleicher, 1997). Os fungicidas mais eficientes usados no controle químico de Monilinia fructicola em pêssegos, na pós-colheita, segundo Andrade \& Matos (1996), foram iprodione, vinclozolin e triforine, enquanto benomil, thiabendazole, benomil + captan e thiabendazole + captan não controlaram o fungo, resultando em índice de doença semelhante ao da testemunha.

A redução das perdas pós-colheita, causadas por microrganismos, na cadeia produtiva de frutas, representa um grande desafio, pois o uso de fungicidas químicos ainda é um dos métodos mais utilizados. Entretanto, há uma grande limitação sobre aspectos de segurança dos mesmos em função dos possíveis efeitos carcinogênicos e teratogênicos, bem como toxicidade residual (Skandamis et. al., 2001; Eckert et al., 1994). Além disso, a produção de frutos de qualidade, garantindo menor número de uso de agroquímicos e menor efeito sobre o homem e o meio ambiente, é o objetivo da produção integrada, fator fundamental em estratégia de marketing na comercialização (Girardi, 2001).

No controle de doenças, a substituição do uso de agrotóxicos por meios alternativos eficientes é um dos grandes desafios da agricultura sustentável. Bettiol et al. (2005) sugeriram alguns métodos alternativos para o controle de doenças, dentre os quais o controle físico e produtos alternativos, como taninos, extrato de plantas, sais, fosfitos e outros.

A utilização de substâncias naturais torna os produtos mais atrativos ao consumidor por não apresentar efeito tóxico, mesmo quando empregadas em concentrações relativamente elevadas. Além dos benefícios proporcionados à saúde, diversos estudos têm demonstrado o efeito inibidor de produtos naturais sobre o desenvolvimento de microrganismos patogênicos.

Neste contexto, vários estudos têm envolvido a atividade inibidora dos óleos essenciais e extrato de cravo sobre fungos (Hitokoto et al., 1980; Azzouz \& Bullerman, 1982; Shelef, 1983). O cravoda-índia (Caryophillus aromaticus L.), devido a sua origem, contém de 14 a $20 \%$ de óleo volátil nos botões florais secos, sendo constituído de eugenol (70 a 95\%), acetato de eugenol e á-cariofileno (5 a $8 \%$ ). Ele tem ação germicida, antisséptica, desinfetante e anestésica local (Araújo, 2005). O cravo em pó causou inibição de $100 \%$ do desenvolvimento micelial dos fungos Aspergillus niger, Rhizopus spp., Eurotrium repus e Penicillium spp. (Pereira, 2001).

Entre os produtos menos tóxicos que são muito utilizados no processo de sanitização de frutos na fase pós-colheita, estão as soluções cloradas. $\mathrm{O}$ dióxido de cloro tem sido citado como alternativa ao uso de hipocloritos para sanitização de frutos.

O dióxido de cloro é um gás recentemente aprovado como agente antimicrobiano e que tem ação rápida sobre largo espectro de microrganismos 
(Oliveira \& Oliveira, 2004). O dióxido de cloro a 2 e 3 $\mathrm{mL} / \mathrm{L}$ e o cloreto de benzalcônio a $1 ; 2$ e $3 \mathrm{~mL} / \mathrm{L}$, aplicados de forma curativa, inibiram a incidência (\% de frutos doentes) de Monilinia fructicola e inoculada sem ferimentos e não tiveram eficiência sobre Rhizopus spp. (Abreu, 2006).

$\mathrm{O}$ dióxido de cloro $\left(\mathrm{ClO}_{2}\right)$ formulado comercialmente é uma alternativa para o controle de Botrytis cinerea, Penicillium expansum e Rhizopus stolonifer em pós-colheita (Zoffoli et al., 2005).

Dessa forma, este trabalho teve como objetivo pesquisar produtos alternativos, aplicados na pós-colheita, no controle da podridão-parda (Monilinia fructicola) e da podridão-mole (Rhizopus spp.) em pêssegos visando a preservar a qualidade final do produto com benefícios para o ambiente e para o homem.

\section{MATERIAL E MÉTODOS}

Os frutos para condução do experimento foram colhidos em talhão de pessegueiro da cultivar Diamante, com 10 anos de idade, no município de Nepomuceno-MG. O delineamento experimental utilizado foi o inteiramente casualizado, com 7 tratamentos e 3 repetições.

Para estudar o efeito pós-colheita dos tratamentos alternativos em pêssego, foram selecionados frutos sem qualquer tipo de lesões e sem sintomas de doenças, de área não tratada na pré-colheita, os quais foram encaminhados ao laboratório, onde foi feita uma segunda seleção. Estes foram separados em parcelas para a aplicação dos tratamentos. Os frutos de cada parcela foram mergulhados por 30 segundos em solução de 10 litros de água, contendo os seguintes tratamentos nas concentrações: 1 - Óleo de cravo $0,01 \%$; 2 Dióxido de cloro 0,1\%; 3- Dióxido de cloro 0,05\%; 4Cloreto de benzalcônio $0,25 \%$; 5 - Dicloran $0,12 \%$; 6Iprodione $0,15 \%$ e 7 - Testemunha, sem tratamento químico. Após este procedimento, os frutos de cada tratamento foram deixados em repouso para escorrer o excesso de calda sobre papel toalha e, em seguida, os mesmos foram colocados em bandejas de plástico também forradas com papel toalha, todo material foi esterilizado. O experimento foi conduzido em condições de ambiente, no laboratório, em dois anos consecutivos. As parcelas foram constituídas de 10 frutos por bandeja em 2005 e 8 frutos em 2006, contendo três repetições cada.

Foram avaliadas a incidência e a severidade das doenças aos 3 e 5 dias após o início do armazenamento, em 2005, e aos 3; 6 e 9 dias em 2006.
Para avaliar a severidade, foram dadas notas às lesões da seguinte maneira: 1 - até $25 \%$ do fruto com lesão; 2- 26 a $50 \%$; 3- 51 a $75 \%$ e $4->76 \%$. Avaliou-se a severidade em cada fruto, calculando-se a média das notas obtidas por parcela e transformando em porcentagem de área afetada. Os dados da porcentagem de incidência e severidade foram transformados em arc-seno $\sqrt{\mathrm{x}}$.

A análise de variância foi realizada de acordo com o modelo usual inteiramente casualizado, em esquema de parcelas subdivididas no tempo, para as variáveis estudadas. A comparação entre as médias dos diferentes tratamentos efetuou-se pelo teste de Scott-Knott, ao nível de 5\% de probabilidade, utilizando-se do software SISVAR (Ferreira, 2000).

\section{RESULTADOS E DISCUSSÃO}

Observou-se pelos resultados que, no primeiro ano de avaliação (2005), prevalesceu a ocorrência de Monilinia fructicola (Tabela 1), e no segundo ano (2006), a de Rhizopus spp.(Tabela 2). Essa ocorrência foi natural, provavelmente influenciada pelo ambiente.

A incidência média da podridão-parda foi significativamente menor nos frutos que foram submetidos ao tratamento com iprodione, que manteve quase todos os frutos sem sintomas da doença até o quinto dia após o tratamento (Tabela 1). O iprodione é um fungicida de uso comum na fruticultura com bons resultados no controle da podridão-parda, conforme verificado nos trabalhos de Feliciano \& Sachs (1984), Bleicher (1997) e Fortes (2003).

O óleo de cravo, dióxido de cloro e cloreto de benzalcônio usados no tratamento pós-colheita dos frutos de pêssego, nas dosagens e condições em que foram conduzidos os experimentos, não reduziram a incidência da podridão-parda (Tabela 1). No entanto, trabalhos de Abreu (2006), com 2 e 3 mL/ $\mathrm{L}$ de dióxido de cloro e 1; 2 e $3 \mathrm{~mL} / \mathrm{L}$ de cloreto de benzalcônio mostraram atividade curativa, reduzindo a incidência de $M$. fructicola inoculada em pêssegos sem ferimentos.

Com relação à severidade da podridão-parda nos frutos, os resultados obtidos foram semelhantes aos verificados nas avaliações da incidência (Tabela 1), onde o iprodione reduziu significativamente a severidade da doença e o produto à base de dicloran com eficiência intermediária.

Na repetição do experimento, segundo ano, a ocorrência predominante foi a podridão de Rhizopus spp., que foi avaliada aos 3; 6 e 9 dias. Os resultados das avaliações de incidência da doença, aos seis e 
nove dias, revelaram que os tratamentos com óleo de cravo $(0,01 \%)$ e dióxido de cloro $0,1 \%$ reduziram significativamente a incidência de Rhizopus spp., juntamente com os produtos dicloran e iprodione (Tabela 2).

Vários trabalhos relatam a atividade inibidora do óleo e do extrato de cravo sobre diversos microrganismos (Hitokoto et al., 1980; Azzous \& Bullerman, 1982; Shelef,1983; Araújo, 2005). Pereira (2001) concluiu que o cravo em pó causou inibição de $100 \%$ no desenvolvimento micelial de Rhizopus spp. No presente estudo, o óleo de cravo mostrou ser promissor no controle do Rhizopus spp., quando comparado aos fungicidas químicos usados em fruticultura. Outras doses e métodos de aplicações devem ser estudados.
O dióxido de cloro formulado comercialmente é uma alternativa para o controle do Rhyzopus stolonifer em pós-colheita conforme Zoffoli et al. (2005). No entanto, o produto não teve eficiência no controle do patógeno nas dosagens de 2 e $3 \mathrm{~mL} / \mathrm{L}$, segundo resultados obtidos por Abreu (2006).

Avaliando a severidade da podridão-mole nos frutos (Tabela 2), verificou-se que, aos seis dias após os tratamentos, o óleo de cravo, dióxido de cloro, iprodione e o dicloran mantiveram a porcentagem da área dos frutos atacada abaixo dos demais tratamentos e da testemunha. No entanto, aos nove dias, com frutos em estágio bem avançado da podridão, apenas os tratamentos com dicloran e iprodione mantiveram a severidade significativamente baixa. Isso se deve provavelmente ao maior poder residual desses produtos em relação ao óleo de cravo e dióxido de cloro.

TABELA 1 - Incidência e severidade da podridão-parda em frutos de pessegueiro, aos 3 e 5 dias após diferentes tratamentos pós-colheita em 2005.

\begin{tabular}{|c|c|c|c|c|}
\hline \multirow[t]{2}{*}{ Tratamentos } & \multicolumn{2}{|c|}{ Incidência $(\%)$} & \multicolumn{2}{|c|}{ Severidade $(\%)$} \\
\hline & (3 dias) & (5 dias) & (3 dias) & (5 dias) \\
\hline 1-Óleo de cravo & $43,3 \mathrm{c}$ & $70,0 \mathrm{c}$ & $11,66 \mathrm{c}$ & $42,66 \mathrm{c}$ \\
\hline 2-Dióxido de cloro & $23,3 \mathrm{~b}$ & $70,0 \mathrm{c}$ & $6,00 \mathrm{c}$ & $38,61 \mathrm{c}$ \\
\hline 3-Dióxido de cloro & $30,0 \mathrm{~b}$ & $66,6 \mathrm{c}$ & $8,33 \mathrm{c}$ & $46,66 \mathrm{c}$ \\
\hline 4-Cloreto de benzalcônio & $40,0 \mathrm{c}$ & $70,0 \mathrm{c}$ & $13,66 \mathrm{c}$ & $46,33 c$ \\
\hline 5-Dicloran & $10,0 \mathrm{a}$ & $43,3 \mathrm{~b}$ & $3,00 \quad b$ & $12,66 b$ \\
\hline 6-Iprodione & $0,00 \mathrm{a}$ & $13,3 \mathrm{a}$ & 0,00 & $5,33 \mathrm{a}$ \\
\hline 7-Testemunha & $26,6 \mathrm{~b}$ & $60,0 \mathrm{c}$ & 7,66 & $37,00 \mathrm{c}$ \\
\hline CV (\%) & 21,23 & 21,23 & 14,27 & 14,27 \\
\hline
\end{tabular}

Médias seguidas por letras distintas nas colunas diferem entre si, pelo teste Scott Knott-5\%.

Dados transformados em arc-seno $\sqrt{\mathrm{x}}$.

TABELA 2 - Incidência e severidade da podridão-mole em frutos de pessegueiro, aos 3, 6 e 9 dias após diferentes tratamentos pós-colheita em 2006.

\begin{tabular}{|c|c|c|c|c|c|c|}
\hline \multirow[t]{2}{*}{ Tratamentos } & \multicolumn{3}{|c|}{ Incidência (\%) } & \multicolumn{3}{|c|}{ Severidade $(\%)$} \\
\hline & (3 dias) & (6 dias) & (9 dias) & (3 dias) & (6 dias) & (9 dias) \\
\hline 1-Óleo de cravo & $8,25 \mathrm{a}$ & $12,5 \mathrm{a}$ & $58,25 \mathrm{a}$ & 6,66 a & $10,66 \mathrm{a}$ & $37,66 \mathrm{~b}$ \\
\hline 2-Dióxido de cloro & $4,13 \mathrm{a}$ & $20,75 \mathrm{a}$ & $62,50 \mathrm{a}$ & $1,00 \mathrm{a}$ & $12,00 \mathrm{a}$ & $49,00 \mathrm{c}$ \\
\hline 3-Dióxido de cloro & $4,13 \mathrm{a}$ & $33,25 \mathrm{~b}$ & $75,00 \mathrm{~b}$ & $2,00 \mathrm{a}$ & $17,77 \mathrm{~b}$ & $50,00 \mathrm{c}$ \\
\hline 4-Cloreto de benzalcônio & 8,25 a & $41,63 \mathrm{~b}$ & $83,25 \mathrm{~b}$ & $2,00 \mathrm{a}$ & $25,00 \mathrm{~b}$ & $57,66 \mathrm{c}$ \\
\hline 5-Dicloran & $0,00 \mathrm{a}$ & $0,00 \mathrm{a}$ & $41,63 \mathrm{a}$ & $0,00 \mathrm{a}$ & $0,00 \mathrm{a}$ & $15,66 \mathrm{a}$ \\
\hline 6-Iprodione & $4,13 \mathrm{a}$ & $16,63 \mathrm{a}$ & $50,00 \mathrm{a}$ & $1,00 \mathrm{a}$ & $10,66 \mathrm{a}$ & $26,33 \mathrm{a}$ \\
\hline 7-Testemunha & $12,50 \mathrm{a}$ & $29,13 \mathrm{~b}$ & $70,75 \mathrm{~b}$ & 6,33 a & $20,66 \mathrm{~b}$ & $56,66 \mathrm{c}$ \\
\hline $\mathrm{CV}(\%)$ & 27,41 & 27,41 & 27,41 & 18,70 & 18,70 & 18,70 \\
\hline
\end{tabular}

Médias seguidas por letras distintas nas colunas diferem entre si, pelo teste Scott Knott-5\%.

Dados transformados em arc-seno $\sqrt{\mathrm{x}}$. 


\section{CONCLUSÕES}

1-O iprodione aplicado em pós-colheita controlou a incidência e a severidade de $M$. fructicola e de Rhizopus spp. em pêssegos.

$2-\mathrm{O}$ dicloran a $0,12 \%$ foi o tratamento mais eficiente para o controle de Rhizopus spp. e intermediário para $M$. fructicola, reduzindo significativamente a severidade dessas podridões em pêssegos.

3-Os tratamentos com óleo de cravo a $0,01 \%$, e dióxido de cloro na dose de $0,1 \%$ reduziram a incidência de Rhizopus spp. até o nono dia, e a severidade, até o sexto dia após o tratamento.

\section{AGRADECIMENTOS} pesquisa.

À FAPEMIG, pelo financiamento desta

\section{REFERÊNCIAS}

ABREU, F. M. de. Quantificação de danos e controle pós-colheita de podridão parda (Monilinia fructicola) e podridão mole (Rhizopus spp.) em pêssegos. 2006. 95 F. Dissertação (Mestrado em Fitopatologia) - Escola Superior de Agricultura Luiz de Queiros, Universidade de São Paulo, Piracicaba, 2006.

ALVAREZ, A. M.; NISHIJIMA, W. T. Posthavest diseases of papaya. Plant Disease, St. Paul, v. 71, n. 8, p. 681-686, 1987.

ANDRADE, E. R. de. Doenças do pessegueiro e da ameixeira e seu controle no Estado de Santa Catarina. Florianópolis: EPAGRI, 1995.52 p. (Boletim Técnico, 71).

ANDRADE, E. R.; MATOS, C. S. Controle químico de Monilinia fructicola em pêssego na pós-colheita. Fitopatologia Brasileira, Brasília, v. 21, n. 2, p. 301303, jun. 1996.

ARAUJO, R. de C.Z. Embalagens ativas com ervas aromáticas e condimentares na conservação de pães artesanais. 2005. 88 f. Dissertação (Mestrado em Ciência dos Alimentos) - Universidade Federal de Lavras, Lavras, 2005.

AZZOUZ, M. A.; BULLERMAN, L. B. Comparative antimycotic effects of selected herbs, spices, planta components and commercial anti-fungal agents. Journal of Food Protection, Ames, v. 45, n. 14, p. 1298-1301, 1982.

BENATO, E. A. Controle de doenças pós-colheita em frutas tropicais. Summa Phytopathologica, Jaboticabal, v. 25, n. 1, p. 90-93, 1999.

BETTIOL, W.; GHINI, R.; MORANDI, M. A. B. Alguns métodos alternativos para o controle de doenças de plantas disponíveis no Brasil. In: VENZON, M.; JUNIOR, T.J. DE P.; PALLINE, A. (Coord.). Controle alternativo de pragas e doenças. Viçosa-MG: EPAMIG/CTZM: UFV, 2005. p.163-183.

BLEICHER, J. Doenças de rosáceas de caroço. In: KIMATI, H.; AMORIM, L.; BERGAMIN F.; CAMARGO, L. E. A.; REZENDE, J. A. M. (Ed.). Manual de fitopatologia: doenças das plantas cultivadas. 3.ed. São Paulo: Ceres, 1997. p. 621-627.

ECKERT, J. W.; SIEVERT, J. R.; RATNAYAKE, M. Reduction of imazalil effectiveness against citrus green mold in California packinghouses by resistant biotypes of Penicillium digitatum. Plant Disease, $\mathrm{St}$ Paul, v. 78, p. 791-794, 1994.

FELICIANO, A.; SACHS, S. Doenças. In: EMBRAPA. A cultura do pessegueiro. Pelotas, 1984. p. 89-101. (Circular Técnica, 10).

FERREIRA, D. F. Análises estatísticas por meio do SISVAR para Windows versão 4.0. In: REUNIÃO ANUAL DA REGIÃO BRASILEIRA DA SOCIEDADE INTERNACIONAL DE BIOMÉTRIA, 45., 2000, São Carlos. Programa e resumos... São Carlos: UFSCar, 2000. p. 235.

FORTES, J. F. Principais doenças. In: RASEIRA, M. DO C. B.; CENTELLAS-QUESADA, A. (Ed.). Pêssego: produção. Brasília-DF: Embrapa Informação Tecnológica, 2003. p.107-114 (Frutas do Brasil, 49).

FORTES, J. F.; BETTIOL, W. Controle biológico e químico de Monilinia fructicola, com tratamento pós-colheita de pêssegos. Fitopatologia Brasileira, Brasília, v. 22, p. 264, 1997. Suplemento

GIRARDI, C. L. Manejo pós-colheita e rastreabilidade da fruta na produção integrada. Informe Agropecuário, Belo Horizonte, v. 22, n. 213, p. 75-78, 2001. 
HITOKOTO, H.; MOROZUMI, S.; WAUKE, T.; SAKAI, S.; KURATA, H. Inhibitory effects of spices on growth and toxin production of toxigenic fungi. Tokyo. Applied and Evironmental Microbiology, Washington, v. 39, n. 4, p. 818-822, 1980.

JUNQUEIRA, A. H.; PEETZ, M. S. Aspectos relevantes dos mercados interno e externo. In: RASEIRA, M. DO C. B.; CENTELLAS-QUESADA (Ed.). Pêssego: produção. Brasília-DF: Embrapa Informação Tecnológica, 2003. p. 18-30 (Frutas do Brasil, 49).

MARTINS, M. C.; AMORIM, L. Doenças das rosáceas de caroço. Informe Agropecuário, Belo Horizonte, v. 26, n. 228, p. 44-48, 2005.

OLIVEIRA, L. M. de.; OLIVEIRA, P. A. P. L. V. de. Revisão: Principais agentes antimicrobianos utilizados em embalagens plásticas. Brazilian Journal of Food Technology, Campinas, v. 7, n. 2, p. 161-165, 2004.

PEREIRA, M. C. Efeito da adição de condimentos no controle de microrganismos, na conservação de produtos de panificação e na inibição de metabólitos produzidos por fungos associados ao café. 2001. 104 f. Dissertação (Mestrado em Ciências dos Alimentos) - Universidade Federal de Lavras, Lavras, 2001.
SHELEF, L. A. Antimicrobial effects os spices. Journal of Food Safety, Connecticut, v. 6, n. 1, p. 2944, 1983.

SKANDAMIS, P.; KOUTSOUMANIS, K.; FASSEAS, K.; \& NYCHAS, G. J. E. Inhibition of oregano essential oil and EDTA on E.coli 0157:H7. Italian Journal of Food Science, Roma, v. 13, p. 55$65,2001$.

WILSON, C.L.; EL GHAOUTH, A.; CHALUTZ, E.; DROBY, S.; STEVENS, C.; LU, J.Y.; KHAN, V.; ARUAL, J. Potential of induced resistence to control postharvest diseases of fruits and vegetables. Plant diseases, St. Paul, v. 78, n. 9, p. 837-844, 1994.

ZOFFOLI, J. P.; LATORRE, B. A.; DAIRE, N.; VIERTEL, S. Efectividad del Dióxido de Cloro, en Función de la Concentración, pH y Tiempo de Exposición, em el Control de Botrytis cinerea, Penicillium expansum y Rhizopus stolonifer. Ciencia e Investigación Agrária, Santiago, v. 32, n. 3, p. 181-188, 2005. 